\title{
Why the GMC should set up a central registry of doctors' competing interests
}

We omitted one person from the list of signatories in this Open Letter to the General Medical Council (BMJ 2014;348:g236, doi:10.1136/bmj.g236). It should also have included Martin

Brunet, general practitioner, Binscombe Medical Centre,

Godalming, Surrey. 